\title{
Yield and Yield Contributing Performance of 75 Tomato Germplasm in Bangladesh
}

\author{
Ferdouse Islam, AKM Quamruzzaman* ${ }^{\circledR}$, Sharmilla Rani Mallick \\ Olericulture Division, Horticulture Research Center, Bangladesh Agricultural Research Institute, Gazipur, Bangladesh \\ Email: *akmqzs@gmail.com
}

How to cite this paper: Islam, F., Quamruzzaman, AKM and Mallick, S.R. (2021) Yield and Yield Contributing Performance of 75 Tomato Germplasm in Bangladesh. Agricultural Sciences, 12, 1295-1306. https://doi.org/10.4236/as.2021.1211083

Received: September 25, 2021

Accepted: November 15, 2021

Published: November 18, 2021

Copyright $\odot 2021$ by author(s) and Scientific Research Publishing Inc. This work is licensed under the Creative Commons Attribution-NonCommercial International License (CC BY-NC 4.0). http://creativecommons.org/licenses/by-nc/4.0/ (c) (i) (s) Open Access

\begin{abstract}
The study was conducted with 75 tomato germplasm/lines at the farm of Olericulture Division, Horticulture Research Centre (HRC), Bangladesh Agricultural Research Institute, Gazipur, Bangladesh during the winter season of 2020-21 to evaluate the WorldVeg tomato germplasm. The days to first harvest were varied from 106 to 116 days while twelve lines were harvested within 106 - 108 days. The number of fruits per plant varied from 11.7 to 71.3 , while the range of single fruit weight was $16.4-186.6 \mathrm{~g}$. Fruit yield per hectare was 15.7 - $150.4 \mathrm{t} / \mathrm{ha}$ where the highest fruit yield per hectare was obtained from the lines AVTO 1702 (150.40 t/ha), followed by AVTO 1409 (126.54 t/ha), AVTO 1010 (120.60 t/ha), AVTO 1712 (111.33 t/ha), AVTO 1711 (107.23 $\mathrm{t} / \mathrm{ha}$ ). The range of fruit length was observed 2.07 to $6.8 \mathrm{~cm}$, while the fruit diameter was 1.9 to $7.4 \mathrm{~cm}$. The range of pericarp thickness was 0.14 to 0.8 $\mathrm{cm}$, while the range of number of locule was 2.0 to 8.2 . The range of TSS level was $2.0 \%-8.2 \%$, while the shelf life of the tomato lines was recorded 5.2 to 10.3 days. Considering earliness, quantitative and qualitative characters, eleven WorldVeg Center tomato lines AVTO 1010, AVTO 1315, AVTO 1409, AVTO 1711, AVTO 1712, AVTO 1713, AVTO 1717, AVTO 1907, AVTO 1911, AVTO 1915 and AVTO 1921 were found promising. So, these eleven lines can be selected for further confirmation.
\end{abstract}

\section{Keywords}

Yield, Flower, Fruit Characters, Performance, Tomato Germplasm, Bangladesh

\section{Introduction}

Tomato (Solanum lycopersicum) is one of the most important vegetables of Bangladesh and the world wide, too. It is the second most important world consumable vegetable after potato, ranks first among the processing crops [1] and 
belongs to Solanaceae family [2]. It is rich in a plethora of natural antioxidants and bioactive compounds. The regular ingestion of an adequate amount of fresh tomatoes or processed tomato products has been inversely correlated with the development of widespread human diseases [3] [4] [5] and with an increase in plasma lipid peroxidation levels [6] [7]. This protective effect has been mainly attributed to the carotenoid constituents of the fruits, particularly lycopene and $\beta$-carotene which act as antioxidants in detoxifying free radicals [4] [8] [9] [10]. [11] reported that both fresh and processing tomatoes constitute significant amounts within produced vegetable crops. In addition to the large production values, the tomato is an important source of antioxidant intake. The essential phytonutrients found in the tomato fruit are lycopene, $\beta$-carotene, $\alpha$-tocopherol, polyphenols and ascorbic acid, and antioxidant activity depends on cultivar, environmental parameters, method of production and processing. The ripe tomatoes have important outside quality characteristics: uniform size, red colour and good aroma and texture but these external qualities are not reflecting perfectly the nutritional content.

However, Bangladesh Agricultural Research Institute (BARI) has released 21 open-pollinated (OP) and 11 hybrid tomato varieties so far. Several leading seed companies are also supplying some more tomato varieties and seeds which are being imported from different countries. Though the BARI released varieties are higher yielder, some varieties are prone to several pests and diseases. Beside this growers' demand is good quality summer hybrid varieties. So, growers are interested to get good quality pest and diseases resistant winter and summer tomato varieties having good shelf life. In this context, BARI needs to develop quality tomato varieties having good keeping quality along with higher yield. In 2020, The World Vegetable Centre has supplied 43 tomato advanced lines in two installments. Therefore, an experiment was under taken for assessing horticultural traits and yield potentiality along with local germplasm and varieties in Bangladesh condition to select suitable tomato lines for developing good quality pest and diseases resistant winter and summer tomato varieties having good shelf life.

\section{Materials and Methods}

\subsection{Experimental Site}

The experiment was conducted at the Olericulture Division of Horticulture Research Centre, Bangladesh Agricultural Research Institute (BARI) during $2020-21$. The experimental field was at $23.9920^{\circ} \mathrm{N}$ Latitude and $90.4125^{\circ} \mathrm{E}$ Longitudes having an elevation of $8.2 \mathrm{~m}$ from sea level under agro-ecological zone (AEZ) 28. The average minimum and maximum temperature were $18.0^{\circ} \mathrm{C}$ and $29.0^{\circ} \mathrm{C}$ and the average relative humidity was $58.2 \%$. The soil of the experimental field was sandy clay loam in texture having a $\mathrm{pH}$ range around 6.0.

Air temperatures and relative humidity of the experimental area

Average monthly minimum air temperatures, maximum air temperatures and 
average relative humidity during the season are shown in Table 1.

\subsection{Plant Materials}

Seventy-five tomato germplasm/varieties (WorldVeg Center: 41 nos., BARI varieties: 10 nos., local germplasm 17 nos., exotic germplasm 7 nos.) were included in the study (Table 2). The seeds were sown on the seedbed on 01 October 2020. Thirty-two days old seedlings were transplanted in the main field on 01 November, 2020.

\subsection{Experimental Design and Layout}

The experiment was laid out in a RCB design with three replications. The plot

Table 1. Average monthly minimum temperature $\left({ }^{\circ} \mathrm{C}\right)$, maximum temperature $\left({ }^{\circ} \mathrm{C}\right)$ and relative humidity $(\%)$ recorded during winter season.

\begin{tabular}{cccccccc}
\hline Parameter & Oct. 2020 & Nov. 2020 & Dec. 2020 & Jan. 2021 & Feb. 2021 & Mar. 2021 & Mean \\
\hline Monthly minimum air temperature $\left({ }^{\circ} \mathrm{C}\right)$ & 24 & 19 & 14 & 16 & 15 & 20 & 18.0 \\
Monthly maximum air temperature $\left({ }^{\circ} \mathrm{C}\right)$ & 31 & 29 & 26 & 28 & 28 & 32 & 29.0 \\
Monthly mean relative humidity $(\%)$ & 72 & 66 & 63 & 54 & 49 & 45 & 58.2 \\
\hline
\end{tabular}

Table 2. Seventy-five tomato germplasm/varieties.

\begin{tabular}{|c|c|c|c|c|c|}
\hline \multicolumn{3}{|c|}{ WorldVeg Center germplasm } & \multirow{2}{*}{$\begin{array}{l}\text { BARI varieties } \\
\text { BARI Tomato-2 }\end{array}$} & \multirow{2}{*}{$\begin{array}{c}\text { Local germplasm } \\
\text { SLA } 011\end{array}$} & \multirow{2}{*}{$\begin{array}{c}\text { Exotic germplasm } \\
\text { SLA } 004\end{array}$} \\
\hline AVTO 0301 & AVTO 1706 & AVTO 1911 & & & \\
\hline AVTO 1003 & AVTO 1707 & AVTO 1913 & BARI Tomato-11 & SLA 012 & SLA 005 \\
\hline AVTO 1008 & AVTO 1711 & AVTO 1914 & BARI Tomato-14 & SLA 013 & SLA 006 \\
\hline AVTO 1010 & AVTO 1712 & AVTO 1915 & BARI Tomato-15 & SLA 014 & SLA 007 \\
\hline AVTO 1219 & AVTO 1713 & AVTO 1919 & BARI Tomato-16 & SLA 015-1 & SLA 008 \\
\hline AVTO 1288 & AVTO 1715 & AVTO 1921 & BARI Tomato-17 & SLA 015-2 & SLA 009 \\
\hline AVTO 1306 & AVTO 1716 & AVTO 1954 & BARI Tomato-18 & SLA 015-3 & SLA 010 \\
\hline AVTO 1314 & AVTO 1717 & & BARI Tomato-19 & SLA 018 & \\
\hline AVTO 1315 & AVTO 1718 & & BARI Tomato 20 & SLA 025-1 & \\
\hline AVTO 1409 & AVTO 1719 & & BARI Tomato 21 & SLA $025-2$ & \\
\hline AVTO 1424 & AVTO 1720 & & & SLA 025-3 & \\
\hline AVTO 1429 & AVTO 1828 & & & SLA 025-4 & \\
\hline AVTO 1464 & AVTO 1829 & & & SLA $025-5$ & \\
\hline AVTO 1616 & AVTO 1903 & & & SLA 025-6 & \\
\hline AVTO 1619 & AVTO 1907 & & & SLA 025-7 & \\
\hline AVTO 1702 & AVTO 1909 & & & FOBHT8 & \\
\hline AVTO 1705 & AVTO 1910 & & & МOBHT8 & \\
\hline & 41 & & 10 & 17 & 7 \\
\hline
\end{tabular}


size was $5.0 \times 1.0 \mathrm{~m}$ where 20 plants were planted with space of $60 \times 50 \mathrm{~cm}$ in two rows.

\subsection{Land Fertilization}

The experimental area was enriched with organic fertilizer, Nitrogen, Phosphorus, Potassium, Sulphur, Zinc and Boron @ 3,000, 250, 90, 125, 20, 3 and 2 $\mathrm{kg} / \mathrm{ha}$, correspondingly. One third of the organic fertilizer, 50\% of Phosphorus and full of Sulphur, Zinc and Boron were incorporated for the period of last land-dwelling preparation. Rest of organic fertilizer and Phosphorus and 1/3 of Potassium were applied as basal in pit. Entire quantity of Nitrogen and rest of Potassium were applied in four equal portions beginning from 20 days after transplanting. Rest three portions were fertilized at 20,40 and 60 days after transplanting.

\subsection{Data Recorded}

Data on yield and yield attributing parameters with qualitative traits were recorded from 20 inner plants of each plot escaping border plants following WorldVeg guideline. Tomato fruits were started harvesting at breaker stage from 20 inner plant of each treatment. Four to six harvesting was done according to the different germplasm characters and data on days to first harvest, number of flower cluster per plant, number of fruit per cluster, number of fruit per plant, single fruit weight $(\mathrm{g})$, fruit yield per plant $(\mathrm{kg})$, fruit yield $(\mathrm{t} / \mathrm{ha})$, fruit length $(\mathrm{cm})$, fruit diameter $(\mathrm{cm})$, pericarp thickness $(\mathrm{cm})$, TSS $(\%)$, shelf life, number of locule, number of leaflet, number of compound leaflet, leaf length $(\mathrm{cm})$, leaf diameter $(\mathrm{cm})$, plant height $(\mathrm{cm})$, branches/plant, fruit size, fruit shape, plant growth nature, cluster nature of fruit, type of fruit.

\subsection{Statistical Analysis}

The recorded quantitative data were analyzed statistically and treatments means were compared by Least Significant Difference (LSD) test following R Software 3.1.2 [12]

\section{Results and Discussion}

The tomato germplasm/varieties differed significantly in all parameters studied and the results have been shown in Table 3 and Table 4 . The findings of different parameters revealed that days to first harvest was varied from 106 to 116 days which indicated the variation among the 75 tomato lines in the harvest days. Twelve lines viz., AVTO 1010, AVTO 1315, AVTO 1409, AVTO 1711, AVTO 1712, AVTO 1713, AVTO 1907, AVTO 1911, AVTO 1915, AVTO 1921, AVTO 1954, SLA 025-7 harvested within 106 - 108 days that indicated earliness of the lines. It may be due to the genetic variations among the lines. Number of flower clusters per plant was significantly varied from $11-21$, while number of fruits per cluster counted 3.5 - 9.0. In respect of number of fruits per plant varied 
Table 3. The harvest, flower and fruit characters of 75 tomato lines.

\begin{tabular}{|c|c|c|c|c|c|c|c|c|c|c|c|}
\hline Line & $\begin{array}{c}\text { Days to } \\
\text { first } \\
\text { harvest }\end{array}$ & $\begin{array}{l}\text { No. of } \\
\text { flower } \\
\text { cluster } \\
\text { per plant }\end{array}$ & $\begin{array}{l}\text { No. of } \\
\text { fruit } \\
\text { per } \\
\text { cluster }\end{array}$ & $\begin{array}{l}\text { No. of } \\
\text { fruit } \\
\text { per } \\
\text { plant }\end{array}$ & $\begin{array}{l}\text { Single } \\
\text { fruit } \\
\text { weight } \\
\text { (g) }\end{array}$ & $\begin{array}{c}\text { Fruit } \\
\text { yield per } \\
\text { plant (kg) }\end{array}$ & $\begin{array}{l}\text { Fruit } \\
\text { yield } \\
\text { (t/ha) }\end{array}$ & $\begin{array}{c}\text { Fruit } \\
\text { length } \\
(\mathrm{cm})\end{array}$ & $\begin{array}{c}\text { Fruit } \\
\text { diamete } \\
\text { r (cm) }\end{array}$ & $\begin{array}{l}\text { Pericarp } \\
\text { thickness } \\
(\mathrm{cm})\end{array}$ & $\begin{array}{l}\text { No. of } \\
\text { locule }\end{array}$ \\
\hline AVTO 0301 & 112 & 19 & 4.2 & 30.0 & 60.0 & 1.79 & 59.15 & 4.5 & 4.4 & 0.4 & 4.4 \\
\hline AVTO 1003 & 110 & 15 & 5.7 & 23.3 & 65.0 & 1.51 & 49.76 & 5.5 & 4.0 & 0.6 & 2.6 \\
\hline AVTO 1008 & 113 & 16 & 7.2 & 35.7 & 75.2 & 2.68 & 88.43 & 5.1 & 4.5 & 0.5 & 3.0 \\
\hline AVTO 1010 & 106 & 15 & 7.0 & 41.7 & 88.0 & 3.65 & 120.60 & 5.7 & 5.9 & 0.6 & 3.0 \\
\hline AVTO 1219 & 112 & 15 & 4.3 & 26.7 & 62.3 & 1.67 & 55.22 & 6.3 & 4.6 & 0.7 & 4.8 \\
\hline AVTO 1288 & 112 & 14 & 6.0 & 14.7 & 96.6 & 1.41 & 46.56 & 6.2 & 5.3 & 0.6 & 5.5 \\
\hline AVTO 1306 & 110 & 13 & 8.6 & 24.0 & 74.3 & 1.78 & 58.69 & 4.8 & 3.6 & 0.8 & 5.5 \\
\hline AVTO 1314 & 114 & 14 & 6.4 & 24.3 & 80.0 & 1.92 & 63.46 & 4.9 & 5.2 & 0.3 & 4.7 \\
\hline AVTO 1315 & 108 & 20 & 4.1 & 34.8 & 74.0 & 2.57 & 84.70 & 4.9 & 4.2 & 0.6 & 2.2 \\
\hline AVTO 1409 & 108 & 17 & 5.1 & 44.0 & 87.3 & 3.83 & 126.54 & 5.3 & 5.0 & 0.4 & 3.4 \\
\hline AVTO 1424 & 110 & 14 & 7.0 & 33.7 & 63.7 & 2.14 & 70.48 & 5.1 & 4.6 & 0.6 & 3.0 \\
\hline AVTO 1429 & 111 & 14 & 4.9 & 23.0 & 105.0 & 2.41 & 79.41 & 4.7 & 6.8 & 0.5 & 4.4 \\
\hline AVTO 1464 & 113 & 18 & 5.4 & 21.3 & 102.0 & 2.18 & 71.85 & 5.1 & 5.4 & 0.6 & 6.1 \\
\hline AVTO 1616 & 113 & 17 & 5.1 & 15.3 & 82.6 & 1.26 & 41.67 & 5.0 & 4.9 & 0.6 & 3.3 \\
\hline AVTO 1619 & 111 & 11 & 5.4 & 25.3 & 81.6 & 2.07 & 68.34 & 6.0 & 5.2 & 0.5 & 2.4 \\
\hline AVTO 1702 & 111 & 16 & 7.6 & 27.3 & 186.6 & 4.56 & 150.40 & 5.6 & 5.4 & 0.8 & 2.4 \\
\hline AVTO 1705 & 113 & 15 & 5.0 & 24.3 & 74.7 & 1.82 & 59.95 & 4.5 & 4.3 & 0.4 & 3.0 \\
\hline AVTO 1706 & 114 & 14 & 4.2 & 21.0 & 24.6 & 0.51 & 16.89 & 4.9 & 4.5 & 0.6 & 3.0 \\
\hline AVTO 1707 & 112 & 14 & 6.8 & 19.7 & 74.4 & 1.46 & 48.09 & 5.2 & 4.9 & 0.6 & 3.0 \\
\hline AVTO 1711 & 107 & 17 & 4.7 & 34.7 & 93.7 & 3.25 & 107.23 & 6.2 & 6.7 & 0.6 & 4.8 \\
\hline AVTO 1712 & 107 & 21 & 5.9 & 43.3 & 78.7 & 3.37 & 111.33 & 5.6 & 5.6 & 0.6 & 6.4 \\
\hline AVTO 1713 & 108 & 13 & 8.0 & 30.7 & 87.0 & 2.65 & 87.40 & 6.7 & 5.8 & 0.5 & 4.9 \\
\hline AVTO 1715 & 109 & 15 & 6.2 & 24.0 & 93.0 & 2.23 & 73.64 & 5.6 & 6.5 & 0.6 & 6.8 \\
\hline AVTO 1716 & 112 & 12 & 5.2 & 34.7 & 70.7 & 2.45 & 80.79 & 4.2 & 5.7 & 0.5 & 2.0 \\
\hline AVTO 1717 & 109 & 16 & 5.3 & 36.0 & 83.7 & 3.00 & 98.91 & 5.7 & 6.3 & 0.6 & 5.5 \\
\hline AVTO 1718 & 111 & 13 & 5.5 & 32.7 & 80.0 & 2.61 & 86.16 & 5.2 & 5.3 & 0.6 & 4.7 \\
\hline AVTO 1719 & 113 & 14 & 5.8 & 26.3 & 86.4 & 2.27 & 75.06 & 5.2 & 6.6 & 0.6 & 5.2 \\
\hline AVTO 1720 & 111 & 16 & 3.5 & 24.7 & 85.0 & 2.09 & 69.10 & 5.2 & 6.4 & 0.7 & 5.1 \\
\hline AVTO 1828 & 112 & 18 & 6.4 & 54.7 & 22.0 & 1.21 & 39.92 & 4.5 & 4.2 & 0.3 & 3.3 \\
\hline AVTO 1829 & 111 & 14 & 6.2 & 55.2 & 25.0 & 1.38 & 45.39 & 4.8 & 3.8 & 0.4 & 3.0 \\
\hline AVTO 1903 & 114 & 15 & 5.7 & 31.0 & 74.4 & 2.30 & 76.01 & 5.3 & 5.5 & 0.6 & 3.4 \\
\hline AVTO 1907 & 108 & 16 & 7.0 & 36.7 & 80.7 & 2.95 & 97.38 & 5.1 & 5.4 & 0.7 & 3.3 \\
\hline
\end{tabular}




\section{Continued}

\begin{tabular}{|c|c|c|c|c|c|c|c|c|c|c|c|}
\hline AVTO 1909 & 111 & 15 & 7.0 & 31.0 & 91.4 & 2.83 & 93.27 & 5.9 & 4.8 & 0.4 & 3.0 \\
\hline AVTO 1910 & 113 & 11 & 9.0 & 11.7 & 95.4 & 1.11 & 36.62 & 5.0 & 5.2 & 0.7 & 3.3 \\
\hline AVTO 1911 & 108 & 14 & 5.6 & 30.0 & 87.3 & 2.60 & 85.90 & 5.7 & 6.5 & 0.7 & 8.2 \\
\hline AVTO 1913 & 111 & 15 & 8.0 & 28.3 & 71.3 & 2.01 & 66.31 & 5.7 & 3.6 & 0.7 & 3.0 \\
\hline AVTO 1914 & 115 & 15 & 6.9 & 42.0 & 54.0 & 2.26 & 74.73 & 5.1 & 4.8 & 0.4 & 3.3 \\
\hline AVTO 1915 & 108 & 15 & 6.0 & 32.7 & 85.1 & 2.77 & 91.30 & 5.6 & 4.9 & 0.5 & 2.8 \\
\hline AVTO 1919 & 113 & 12 & 5.8 & 24.7 & 98.4 & 2.42 & 80.01 & 5.0 & 5.9 & 0.4 & 2.6 \\
\hline AVTO 1921 & 108 & 14 & 5.9 & 32.3 & 88.0 & 2.85 & 94.12 & 5.7 & 6.5 & 0.4 & 5.4 \\
\hline AVTO 1954 & 107 & 13 & 5.9 & 28.0 & 90.0 & 2.52 & 83.04 & 5.5 & 5.9 & 0.7 & 2.2 \\
\hline SLA 004 & 109 & 14 & 4.8 & 49.3 & 19.0 & 0.94 & 30.89 & 2.3 & 2.6 & 0.6 & 2.4 \\
\hline SLA 005 & 109 & 16 & 5.4 & 55.0 & 20.4 & 1.12 & 36.90 & 2.6 & 2.8 & 0.2 & 2.0 \\
\hline SLA 006 & 112 & 18 & 6.2 & 48.3 & 23.0 & 1.11 & 36.55 & 2.7 & 2.9 & 0.3 & 2.0 \\
\hline SLA 007 & 110 & 14 & 7.0 & 25.7 & 23.0 & 0.59 & 19.44 & 5.5 & 5.3 & 0.1 & 3.3 \\
\hline SLA 008 & 112 & 12 & 8.8 & 19.0 & 25.3 & 0.48 & 15.74 & 2.7 & 2.9 & 0.3 & 2.4 \\
\hline SLA 009 & 114 & 11 & 5.1 & 22.0 & 50.6 & 1.11 & 36.69 & 3.9 & 4.5 & 0.2 & 4.2 \\
\hline SLA 010 & 113 & 16 & 7.0 & 13.0 & 126.7 & 1.58 & 52.01 & 4.7 & 5.7 & 0.5 & 4.2 \\
\hline SLA 011 & 114 & 15 & 5.0 & 57.7 & 17.4 & 1.01 & 33.19 & 3.3 & 2.4 & 0.3 & 3.0 \\
\hline SLA 012 & 109 & 15 & 6.3 & 52.3 & 17.3 & 0.91 & 30.18 & 2.1 & 2.1 & 0.2 & 2.4 \\
\hline SLA 013 & 110 & 15 & 5.7 & 71.3 & 16.4 & 1.18 & 38.88 & 2.6 & 3.2 & 0.2 & 3.3 \\
\hline SLA 014 & 111 & 13 & 5.9 & 15.7 & 64.4 & 1.01 & 33.18 & 4.7 & 5.2 & 0.5 & 4.2 \\
\hline SLA 015-1 & 109 & 13 & 5.7 & 24.7 & 40.7 & 1.00 & 33.01 & 4.8 & 5.5 & 0.3 & 3.8 \\
\hline SLA 015-2 & 114 & 14 & 6.3 & 32.3 & 44.0 & 1.42 & 46.93 & 6.8 & 3.2 & 0.2 & 3.3 \\
\hline SLA 015-3 & 109 & 15 & 7.0 & 22.0 & 43.4 & 0.95 & 31.34 & 3.5 & 4.3 & 0.3 & 3.8 \\
\hline SLA 018 & 113 & 14 & 4.7 & 17.3 & 60.4 & 1.04 & 34.46 & 5.3 & 3.7 & 0.2 & 3.8 \\
\hline SLA 025-1 & 110 & 12 & 6.2 & 32.3 & 35.0 & 1.13 & 37.30 & 3.3 & 3.2 & 0.3 & 3.4 \\
\hline SLA $025-2$ & 109 & 15 & 6.1 & 38.0 & 32.4 & 1.23 & 40.46 & 5.1 & 3.2 & 0.3 & 3.5 \\
\hline SLA 025-3 & 115 & 17 & 5.2 & 26.0 & 40.0 & 1.04 & 34.20 & 3.4 & 4.6 & 0.3 & 3.5 \\
\hline SLA 025-4 & 114 & 14 & 6.4 & 24.0 & 29.4 & 0.71 & 23.40 & 3.0 & 3.4 & 0.3 & 3.4 \\
\hline SLA $025-5$ & 116 & 12 & 5.7 & 22.0 & 37.0 & 0.82 & 26.96 & 3.3 & 3.2 & 0.3 & 3.6 \\
\hline SLA 025-6 & 113 & 11 & 5.5 & 33.0 & 45.0 & 1.48 & 48.89 & 4.7 & 3.2 & 0.3 & 3.4 \\
\hline SLA 025-7 & 108 & 15 & 5.7 & 34.7 & 48.0 & 1.66 & 54.83 & 4.8 & 3.3 & 0.3 & 3.6 \\
\hline FOBHT8 & 111 & 12 & 5.8 & 34.3 & 62.4 & 2.14 & 70.66 & 5.3 & 5.1 & 0.4 & 3.1 \\
\hline МOBHT8 & 113 & 13 & 4.6 & 35.7 & 65.3 & 2.33 & 76.98 & 4.9 & 5.0 & 0.4 & 3.0 \\
\hline BARI Tomato-2 & 112 & 13 & 5.5 & 32.7 & 82.4 & 2.69 & 88.90 & 5.4 & 5.1 & 0.3 & 3.1 \\
\hline BARI Tomato-11 & 114 & 15 & 5.3 & 52.7 & 22.0 & 1.16 & 38.36 & 2.3 & 1.9 & 0.3 & 2.4 \\
\hline
\end{tabular}




\section{Continued}

\begin{tabular}{ccccccccccccc}
\hline BARI Tomato-14 & 111 & 12 & 4.2 & 31.0 & 89.0 & 2.76 & 90.93 & 5.0 & 5.6 & 0.5 & 3.8 \\
BARI Tomato-15 & 109 & 15 & 6.1 & 27.7 & 91.7 & 2.53 & 83.58 & 5.3 & 5.6 & 0.6 & 3.8 \\
BARI Tomato-16 & 110 & 15 & 5.6 & 31.7 & 78.0 & 2.47 & 81.43 & 4.8 & 4.5 & 0.5 & 2.4 \\
BARI Tomato-17 & 114 & 17 & 5.3 & 14.7 & 158.0 & 2.31 & 76.38 & 5.4 & 7.4 & 0.5 & 4.2 \\
BARI Tomato-18 & 109 & 13 & 6.9 & 32.3 & 79.2 & 2.56 & 84.46 & 5.2 & 5.1 & 0.4 & 2.5 \\
BARI Tomato-19 & 109 & 17 & 6.2 & 31.7 & 75.0 & 2.37 & 78.29 & 4.5 & 5.3 & 0.6 & 3.2 \\
BARI Tomato 20 & 109 & 12 & 5.2 & 46.0 & 25.4 & 1.16 & 38.34 & 3.3 & 3.0 & 0.3 & 2.3 \\
BARI Tomato 21 & 111 & 17 & 4.4 & 31.7 & 77.0 & 2.44 & 80.38 & 5.5 & 5.2 & 0.4 & 3.0 \\
$\quad 10$ & & & $11.7-$ & $16.4-$ & & $15.7-$ & $2.07-$ & $1.85-$ & $0.14-0.8$ & $2.0-8.2$ \\
Range & $106-$ & $11-21$ & $3.5-9.0$ & 71.3 & 186.6 & $0.47-4.6$ & 150.4 & 6.8 & 7.4 & & \\
Mean & 111 & 15 & 5.9 & 31.6 & 67.2 & 1.9 & 63.7 & 4.8 & 4.7 & 0.5 & 3.6 \\
\hline
\end{tabular}

Table 4. Leaf, plant height, branches, TSS and shelf life characters of 75 tomato lines.

\begin{tabular}{|c|c|c|c|c|c|c|c|c|}
\hline Line & $\begin{array}{c}\text { Number of } \\
\text { leaflets }\end{array}$ & $\begin{array}{c}\text { Number of } \\
\text { compound } \\
\text { leaflet }\end{array}$ & $\begin{array}{l}\text { Leaf length } \\
(\mathrm{cm})\end{array}$ & $\begin{array}{l}\text { Leaf } \\
\text { diameter } \\
(\mathrm{cm})\end{array}$ & $\begin{array}{c}\text { Plant height at } \\
\text { last harvest } \\
(\mathrm{cm})\end{array}$ & $\begin{array}{l}\text { Branches/ } \\
\text { plant }\end{array}$ & TSS (\%) & Shelf life \\
\hline AVTO 0301 & 5.0 & 4.0 & 22.9 & 15.2 & 132 & 7.4 & 3.2 & 5.2 \\
\hline AVTO 1003 & 7.0 & 2.5 & 24.9 & 24.9 & 177 & 5.5 & 3.0 & 8.2 \\
\hline AVTO 1008 & 4.3 & 5.9 & 27.3 & 26.9 & 177 & 8.2 & 2.8 & 9.2 \\
\hline AVTO 1010 & 6.1 & 6.5 & 15.9 & 28.9 & 167 & 5.6 & 3.4 & 8.2 \\
\hline AVTO 1219 & 6.5 & 4.9 & 7.3 & 4.4 & 132 & 7.5 & 4.0 & 5.2 \\
\hline AVTO 1288 & 8.5 & 5.8 & 12.2 & 12.6 & 118 & 9.8 & 5.4 & 6.2 \\
\hline AVTO 1306 & 13.5 & 4.5 & 11.9 & 7.9 & 102 & 7.6 & 4.3 & 6.2 \\
\hline AVTO 1314 & 6.6 & 3.8 & 12.7 & 6.4 & 151 & 6.8 & 4.5 & 6.2 \\
\hline AVTO 1315 & 10.5 & 6.0 & 12.2 & 10.6 & 159 & 7.0 & 4.6 & 8.2 \\
\hline AVTO 1409 & 8.6 & 4.9 & 12.4 & 8.9 & 148 & 8.8 & 3.5 & 8.2 \\
\hline AVTO 1424 & 4.6 & 7.0 & 29.9 & 26.4 & 15 & 5.6 & 2.2 & 5.3 \\
\hline AVTO 1429 & 8.0 & 4.4 & 11.2 & 7.2 & 258 & 7.9 & 5.4 & 6.0 \\
\hline AVTO 1464 & 4.5 & 4.0 & 9.7 & 8.2 & 177 & 12.0 & 5.0 & 6.3 \\
\hline AVTO 1616 & 4.9 & 6.4 & 24.9 & 16.0 & 130 & 9.7 & 3.4 & 5.3 \\
\hline AVTO 1619 & 4.6 & 6.6 & 27.8 & 26.4 & 132 & 6.8 & 3.0 & 8.3 \\
\hline AVTO 1702 & 9.5 & 4.0 & 8.1 & 6.7 & 150 & 10.0 & 4.7 & 7.0 \\
\hline AVTO 1705 & 4.3 & 6.4 & 26.7 & 26.9 & 132 & 7.1 & 2.8 & 6.2 \\
\hline AVTO 1706 & 8.0 & 4.3 & 9.4 & 7.2 & 116 & 8.9 & 4.4 & 6.0 \\
\hline AVTO 1707 & 7.0 & 6.0 & 26.6 & 24.4 & 140 & 8.8 & 5.3 & 5.3 \\
\hline AVTO 1711 & 8.7 & 4.0 & 10.2 & 7.4 & 120 & 9.0 & 0.6 & 8.0 \\
\hline
\end{tabular}




\section{Continued}

\begin{tabular}{|c|c|c|c|c|c|c|c|c|}
\hline AVTO 1712 & 9.5 & 4.0 & 10.2 & 7.4 & 121 & 10.2 & 4.4 & 8.3 \\
\hline AVTO 1713 & 6.0 & 7.0 & 28.7 & 26.9 & 155 & 6.6 & 2.2 & 7.3 \\
\hline AVTO 1715 & 4.3 & 5.8 & 29.5 & 31.4 & 146 & 6.8 & 2.0 & 8.2 \\
\hline AVTO 1716 & 3.0 & 6.8 & 25.3 & 22.3 & 150 & 9.1 & 3.0 & 7.3 \\
\hline AVTO 1717 & 10.1 & 4.0 & 12.9 & 10.9 & 135 & 8.3 & 3.0 & 9.2 \\
\hline AVTO 1718 & 4.6 & 10.0 & 13.9 & 10.4 & 131 & 6.0 & 4.4 & 8.0 \\
\hline AVTO 1719 & 4.3 & 6.2 & 23.6 & 22.3 & 132 & 6.8 & 2.4 & 8.2 \\
\hline AVTO 1720 & 3.6 & 6.8 & 26.9 & 21.5 & 128 & 6.8 & 4.3 & 7.3 \\
\hline AVTO 1828 & 4.0 & 5.8 & 26.5 & 25.3 & 238 & 17.6 & 3.8 & 7.3 \\
\hline AVTO 1829 & 4.3 & 6.5 & 24.3 & 22.3 & 140 & 8.3 & 4.4 & 6.2 \\
\hline AVTO 1903 & 4.0 & 6.8 & 29.3 & 25.3 & 151 & 8.3 & 3.6 & 9.2 \\
\hline AVTO 1907 & 10.4 & 5.0 & 33.4 & 27.9 & 127 & 8.8 & 2.5 & 8.2 \\
\hline AVTO 1909 & 9.0 & 4.0 & 26.9 & 22.2 & 114 & 7.6 & 2.7 & 9.2 \\
\hline AVTO 1910 & 5.1 & 6.8 & 24.3 & 18.4 & 116 & 6.6 & 2.5 & 6.2 \\
\hline AVTO 1911 & 5.9 & 4.0 & 23.9 & 15.3 & 407 & 9.8 & 4.0 & 8.2 \\
\hline AVTO 1913 & 6.6 & 4.1 & 24.8 & 24.9 & 238 & 9.5 & 4.4 & 6.2 \\
\hline AVTO 1914 & 7.5 & 9.5 & 7.4 & 8.9 & 121 & 10.9 & 3.5 & 6.3 \\
\hline AVTO 1915 & 3.6 & 5.0 & 17.4 & 20.9 & 155 & 6.2 & 4.0 & 10.3 \\
\hline AVTO 1919 & 5.6 & 7.0 & 28.3 & 25.3 & 128 & 6.8 & 3.0 & 7.2 \\
\hline AVTO 1921 & 8.2 & 3.0 & 10.2 & 7.4 & 135 & 5.4 & 2.5 & 7.2 \\
\hline AVTO 1954 & 4.3 & 6.4 & 29.5 & 26.9 & 149 & 6.9 & 3.0 & 7.2 \\
\hline SLA 004 & 4.0 & 6.3 & 32.4 & 24.6 & 134 & 8.7 & 4.2 & 6.2 \\
\hline SLA 005 & 4.3 & 6.6 & 25.9 & 25.3 & 233 & 6.7 & 5.2 & 5.2 \\
\hline SLA 006 & 9.1 & 3.9 & 10.2 & 7.4 & 244 & 7.1 & 4.6 & 5.2 \\
\hline SLA 007 & 5.6 & 7.0 & 29.6 & 23.9 & 134 & 9.7 & 2.9 & 7.2 \\
\hline SLA 008 & 4.0 & 6.3 & 26.8 & 28.3 & 229 & 7.8 & 4.7 & 6.2 \\
\hline SLA 009 & 3.3 & 6.8 & 27.9 & 21.5 & 67 & 15.0 & 2.2 & 7.2 \\
\hline SLA 010 & 5.3 & 5.3 & 23.6 & 15.2 & 170 & 10.2 & 3.4 & 6.2 \\
\hline SLA 011 & 7.0 & 4.3 & 27.3 & 24.9 & 261 & 6.7 & 5.2 & 7.2 \\
\hline SLA 012 & 4.3 & 6.5 & 25.9 & 21.2 & 106 & 7.1 & 5.0 & 6.2 \\
\hline SLA 013 & 4.3 & 6.5 & 29.5 & 31.4 & 176 & 9.6 & 3.8 & 7.2 \\
\hline SLA 014 & 3.0 & 6.6 & 26.3 & 22.3 & 186 & 7.3 & 3.5 & 6.2 \\
\hline SLA 015-1 & 5.4 & 4.0 & 21.9 & 14.9 & 181 & 5.5 & 2.8 & 5.2 \\
\hline SLA $015-2$ & 4.0 & 3.3 & 24.6 & 15.3 & 169 & 5.2 & 2.9 & 10.2 \\
\hline SLA 015-3 & 4.8 & 4.0 & 21.6 & 18.0 & 174 & 6.2 & 2.7 & 5.2 \\
\hline
\end{tabular}




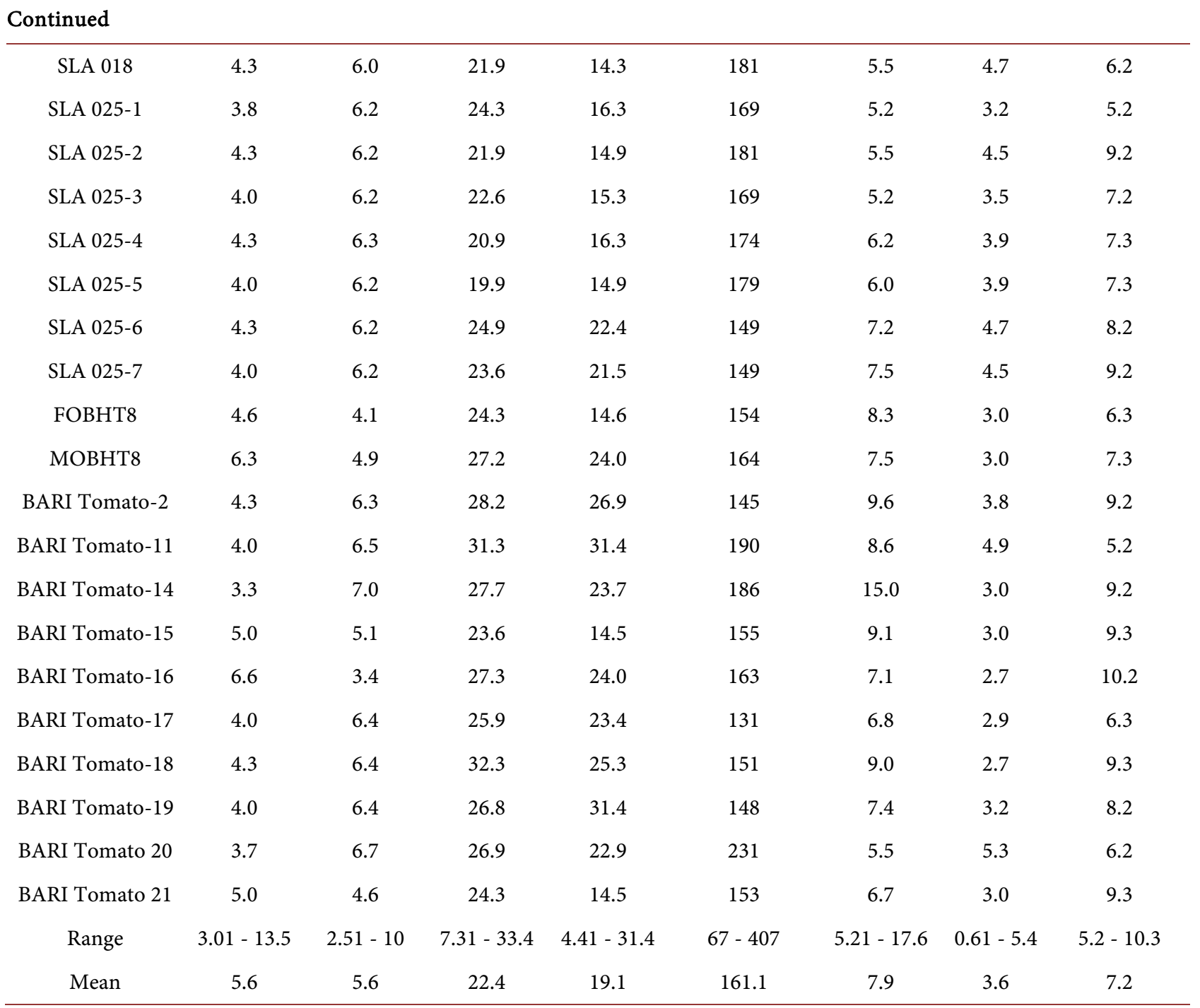

from 11.7 to 71.3, while the highest number of fruit (71.3) was counted from the lines SLA 013 followed by SLA 011 (57.7), SLA 005 (55.0), BARI Tomato-11 (52.7), SLA 012 (52.3), SLA 004 (49.3), SLA 006 (48.3), BARI Tomato 20 (46.0). This high number of fruit bearing is due to smaller sized of fruits. In case of single fruit weight, the range was $16.4-186.6 \mathrm{~g}$, while the largest fruit was harvested from the line AVTO 1702 (186.6 g) followed by BARI Tomato-17(158.0 g), SLA 010 (126.7 g), AVTO 1429 (105.0 g), AVTO 1464 (102.0 g), AVTO 1919 (98.4 g), AVTO 1288 (96.6 g), AVTO 1910 (95.4g), AVTO 1711 (93.7 g), AVTO 1715 $(93.0 \mathrm{~g})$, where the line SLA 013 produced the lowest average fruit weight having $16.4 \mathrm{~g}$. The fruit yield per plant also indicated statistically significant difference which was varied from 0.47 to $4.56 \mathrm{~kg}$, while the line AVTO 1702 exhibited the highest per plant yield (4.56 kg) followed by AVTO 1702 (4.56 kg), AVTO 1409 $(3.83 \mathrm{~kg})$, AVTO $1010(3.65 \mathrm{~kg})$, AVTO $1712(3.37 \mathrm{~kg})$. It was observed that the lines having larger fruit contained were higher yield. Fruit yield per hectare was also varied significantly from 15.7 - 150.4 t/ha. The highest fruit yield per hectare 
was obtained from the lines AVTO 1702 (150.40 t/ha), followed by AVTO 1409 (126.54 t/ha), AVTO 1010 (120.60 t/ha), AVTO 1712 (111.33 t/ha), AVTO 1711 (107.23 t/ha), while the lowest yield (15.74 t/ha) was obtained from the line SLA 008. The low yield was due to less number of fruits as well smaller sizes of fruit.

The fruit length and fruit diameter of different lines varied significantly. The range of fruit length was observed 2.07 to $6.8 \mathrm{~cm}$, while highest fruit length was produced from the line SLA 015-2 $(6.8 \mathrm{~cm})$, followed by AVTO $1713(6.7 \mathrm{~cm})$, AVTO $1288(6.2 \mathrm{~cm})$, AVTO $1619(6.0 \mathrm{~cm})$, AVTO $1909(5.9 \mathrm{~cm})$ and the lowest was produced from SLA $012(2.07 \mathrm{~cm})$. The range of fruit diameter was 1.9 to 7.4 $\mathrm{cm}$, while highest fruit diameter was produced from the line BARI Tomato-17 $(7.4 \mathrm{~cm})$, followed by AVTO $1429(6.8 \mathrm{~cm})$, AVTO $1711(6.7 \mathrm{~cm})$, AVTO 1719 $(6.6 \mathrm{~cm})$, AVTO $1715(6.5 \mathrm{~cm})$, AVTO $1911(6.5 \mathrm{~cm})$, AVTO $1921(6.5 \mathrm{~cm})$, AVTO $1720(6.4 \mathrm{~cm})$, AVTO $1717(6.3 \mathrm{~cm})$, and the lowest diameter was produced from BARI Tomato-11 $(1.9 \mathrm{~cm})$. The pericarp thickness is another important criterion for selecting a good variety. The range of pericarp thickness was 0.14 to $0.8 \mathrm{~cm}$, while the range of number of locule was 2.0 to 8.2 .

Different characters of leaf were studied and observed significant variation among those characters viz., number of leaflets, number of compound leaflet, leaf length, leaf diameter. The range of number of leaflets and number of compound leaflet were counted 3.01 to 13.5 and 2.51 to 10.0 , respectively, while leaf length and leaf diameter were 7.31 to $33.4 \mathrm{~cm}$ and 4.41 to $31.4 \mathrm{~cm}$, respectively. The range of plant height at last harvest significantly varied and it was ranged from 67 to $407 \mathrm{~cm}$. It indicated the variability of growth among the lines, while the range of branches per plant varied from 5.2 to 17.6. Sometime higher TSS (\%) is preferred by some consumers, so it should be categories in different TSS level. The range of TSS level was $2.0 \%-8.2 \%$. The tomato lines were kept under ambient condition (Temp. $26^{\circ} \mathrm{C}-27^{\circ} \mathrm{C}$ ), and the shelf life of the tomato lines was recorded 5.2 to 10.3 days.

\section{Conclusions}

The days to first harvest were varied from 106 to 116 days while twelve lines were harvested within 106 - 108 days. The number of fruits per plant varied from 11.7 to 71.3 , while the range of single fruit weight was $16.4-186.6 \mathrm{~g}$. Fruit yield per hectare was $15.7-150.4 \mathrm{t} /$ ha where the highest fruit yield per hectare was obtained from the lines AVTO 1702 (150.40 t/ha), followed by AVTO 1409 (126.54 t/ha), AVTO 1010 (120.60 t/ha), AVTO 1712 (111.33 t/ha), AVTO 1711 $(107.23 \mathrm{t} / \mathrm{ha})$. The range of fruit length was observed 2.07 to $6.8 \mathrm{~cm}$, while the fruit diameter was 1.9 to $7.4 \mathrm{~cm}$. The range of pericarp thickness was 0.14 to 0.8 $\mathrm{cm}$, while the range of number of locule was 2.0 to 8.2. The range of TSS level was $2.0 \%-8.2 \%$, while the shelf life of the tomato lines was recorded 5.2 to 10.3 days.

Considering earliness, quantitative and qualitative characters, eleven WorldVeg Center tomato lines AVTO 1010, AVTO 1315, AVTO 1409, AVTO 1711, AVTO 
1712, AVTO 1713, AVTO 1717, AVTO 1907, AVTO 1911, AVTO 1915 and AVTO 1921 were found promising. So, these eleven lines can be selected for further confirmation.

\section{Acknowledgements}

This study was completed with the financial support of AFACI under the project of "Development of Vegetable Varieties in Asia Region".

\section{Conflicts of Interest}

The authors declare no conflicts of interest regarding the publication of this paper.

\section{References}

[1] FAO (2008) Tomato Production Statistic. Food and Agriculture Organization of the United Nations, Rome.

[2] Saravanan, S., Thamburaj, S., Veeraragavathatham, D. and Subbiah, A. (2003) Effects of Seaweed Extract and Chlormequat on Growth and Fruit Yield of Tomato (Lycopersicon esculentum Mill.). Indian Journal of Agricultural Research, 37, 79-87.

[3] Agarwal, S. and Rao, A.V. (1998) Tomato Lycopene and Low Density Lipoprotein Oxidation: A Human Dietary Intervention Study. Lipids, 33, 981-984.

https://doi.org/10.1007/s11745-998-0295-6

[4] Erdman, J.W., Ford, N.A. and Lindshield, B.L. (2009) Are the Health Attributes of Lycopene Related to Its Antioxidant Function. Archives of Biochemistry and Biophysics, 483, 229-235. https://doi.org/10.1016/j.abb.2008.10.022

[5] Prakash, A. and Kumar, A. (2014) Implicating the Role of Lycopene in Restoration of Mitochondrial Enzymes and BDNF Levels in $\beta$-Amyloid Induced Alzheimer's Disease. European Journal of Pharmacology, 741, 104-111.

https://doi.org/10.1016/j.ejphar.2014.07.036

[6] Giovannucci, E. (1999) Tomatoes, Tomato-Based Products, Lycopene, and Cancer: Review of the Epidemiologic Literature. Journal of the National Cancer Institute, 91, 317-331. https://doi.org/10.1093/jnci/91.4.317

[7] Balestrieri, M.L., De Prisco, R., Nicolaus, B., Pari, P., Moriello, V.S., Strazzullo, G., Iorio, E.L., Servillo, L. and Balestrieri, C. (2004) Lycopene in Association with R-Tocopherol or Tomato Lipophilic Extracts Enhances Acylplatelet-Activating Factor Biosynthesis in Endothelial Cells during Oxidative Stress. Free Radical Biology and Medicine, 36, 1058-1067. https://doi.org/10.1016/j.freeradbiomed.2004.01.014

[8] Di Mascio, P., Kaiser, S. and Sies, H. (1989) Lycopene as the Most Efficient Biological Carotenoid Singlet Oxygen Quencher. Archives of Biochemistry and Biophysics, 274, 532-538. https://doi.org/10.1016/0003-9861(89)90467-0

[9] Stahl, W. and Sies, H. (1996) Lycopene: A Biologically Important Carotenoid for Humans. Archives of Biochemistry and Biophysics, 336, 1-9. https://doi.org/10.1006/abbi.1996.0525

[10] Clinton, S.K. (1998) Lycopene: Chemistry, Biology and Implications for Human Health and Disease. Nutrition Reviews, 56, 35-51. 
https://doi.org/10.1111/j.1753-4887.1998.tb01691.x

[11] Kotíková, Z., Lachman, J., Hejtmánková, A. and Hejtmánková, K. (2011) Determination of Antioxidant Activity and Antioxidant Content in Tomato Varieties and Evaluation of Mutual Interactions between Antioxidants. LWT-Food Science and Technology, 44, 1703-1710. https://doi.org/10.1016/j.lwt.2011.03.015

[12] Anonymous (2015) R Software.

https://cran.r-project.org/bin/windows/base/ 